Pacific Journal of Mathematic 


\title{
CONGRUENCE SUBGROUPS OF MATRIX GROUPS
}

\author{
IRVING REINER AND J. D. SWIFT
}

1. Introduction. Let $M_{r}^{+}$denote the modular group consisting of all integral $r \times r$ matrices with determinant +1 . Define the subgroup $G_{n}$, of $M_{2}^{+}$to be the group of all matrices

$$
\left(\begin{array}{ll}
a & b \\
c & d
\end{array}\right)
$$

of $M_{2}^{+}$for which $c \equiv 0(\bmod n)$. M. Newman [1] recently established the following theorem:

Let $H$ be a subgroup of $M_{2}^{+}$satisfying $G_{m n} \subset H \subset G_{n}$. Then $H=G_{a n}$, where $a \mid m$.

In this note we indicate two directions in which the theorem may be extended: (i) Letting the elements of the matrices lie in the ring of integers of an algebraic number field, and (ii) Considering matrices of higher order.

2. Ring of algebraic integers. For simplicity, we restrict our attention to the group $G$ of $2 \times 2$ matrices

$$
A=\left(\begin{array}{ll}
a & b \\
c & d
\end{array}\right)
$$

where $a, b, c, d$ lie in the ring $\mathscr{D}$ of algebraic integers in an algebraic number field. Small Roman letters denote elements of $\mathscr{D}$, German letters denote ideals in $\mathscr{D}$.

Let $G(\mathfrak{N})$ be the subgroup of $G$ defined by the condition that $c \equiv 0$ $(\bmod \mathfrak{R})$. We shall prove the following.

THEOREM 1. Let $H$ be a subgroup of $G$ satisfying

$$
G(\mathfrak{M} \Re) \subset H \subset G(\Re),
$$

where $(\mathfrak{M},(6))=(1)$. Then $H=G(\mathfrak{D N})$ for some $\mathfrak{D} \supset \mathfrak{M}$.

Proof. 1. As in Newman's proof, we use induction on the number of prime ideal factors of $\mathfrak{M}$. The result is clear for $\mathfrak{M}=(1)$. Assume it holds for a product of fewer than $k$ prime ideals, and let $\mathfrak{M}=\mathfrak{Q}_{1} \ldots$ $\mathfrak{Q}_{k}(k \geqq 1)$, where the $\mathfrak{\Omega}_{\ell}$ are prime ideals (not necessarily distinct). For

Received June 17, 1955. The research of Professor Swift and the preparation of this paper were supported by the Office of Naval Research under Contract NR 045141. 
the remainder of this section of the proof, let $\Re$ denote a divisor of $\mathfrak{M}$, with $\mathfrak{R} \neq(1)$; set $\mathfrak{M}_{i=\mathfrak{i}} \mathfrak{M} \mathfrak{C}^{\prime}$. Intersecting the groups in (2) with $G(\mathfrak{R} \mathfrak{R})$, we obtain

$$
G(\Re \mathfrak{M} \mathfrak{\Re}) \subset H \cap G(\Re \Re) \subset G(\Re \mathfrak{R})
$$

Since $\mathfrak{M}^{\prime}$ has fewer prime ideal factors than $\mathfrak{M}$, the induction hypothesis gives

$$
H \cap G(\Re \Re)=G\left(\Re^{\prime} \Re \Re\right), \quad \Re^{\prime} \supset \Re^{\prime}
$$

and therefore

$$
G(\Re \Re \Re) \subset H \subset G(\mathfrak{R}) .
$$

Suppose now that for some $\Re$ we have $\Re^{\prime} \neq M^{\prime}$. Then the induction hypothesis yields $H=G(\mathfrak{S N})$ with $\subseteq \supset \Re^{\prime} \mathfrak{\Re} \supset \mathfrak{M}$, and we are through. Thus we may assume hereafter that $\Re^{\prime}=\mathfrak{M}^{\prime}$ for each $\Re$, so that

$$
H \cap G(\mathfrak{R} \mathfrak{R})=G(\mathfrak{M} \mathfrak{R})
$$

for each $\Re$. Therefore for every $A \in H$ given by (1), either $c \in \mathfrak{M} \mathfrak{R}$ or else $((c), \mathfrak{R} \mathfrak{R})=\mathfrak{R}$.

2. Next we shall assume that $H \neq G(\mathfrak{R} \Re)$, and try to establish that $H=G(\Re)$. Let us suppose that $n_{1}, \cdots, n_{t} \in \mathscr{O}$ form a $Z$-basis ${ }^{1}$ for $\mathfrak{R}$, and put

$$
W_{j}=\left(\begin{array}{ll}
1 & 0 \\
n_{j} & 1
\end{array}\right), \quad Y=\left(\begin{array}{ll}
1 & 1 \\
0 & 1
\end{array}\right)
$$

It is easy to see that the $W_{j}$ and the matrix $Y$ generate a complete set of left coset representatives of $G(\mathfrak{R})$ relative to $G(\mathfrak{M} \mathfrak{M})$. Since obviously $Y \in G(\mathfrak{M} \mathfrak{R}) \subset H$, it suffices to prove that each $W_{j} \in H$.

Let us now put $\mathfrak{M}=\prod_{i} \mathfrak{D}_{i}^{a_{i}}, \mathfrak{R}=\prod_{i} \mathfrak{\bigcap}_{i}^{b}$, where $a_{i} \geq 0, b_{i} \geq 0, a_{i}+b_{i}>0$, and $\mathfrak{Q}_{i}, \mathfrak{Q}_{j}$ are distinct prime ideals when $i \neq j$. (Notation: $\left.\mathfrak{a}_{i}^{a} \| \mathfrak{M}\right)$. We shall show that the $Z$-basis $\left\{n_{j}\right\}$ of $\mathfrak{R}$ may be adjusted so that

$$
\mathfrak{D}_{i}^{b} \|\left(n_{j}\right) \quad \text { for each } j \text { and each } i \text {. }
$$

For suppose the $n_{j}$ chosen so that (4) holds for each $j$ for $i=1, \cdots$, $r-1$; we show how to choose a new set $\left\{n_{j}^{\prime}\right\}$ so that $\mathfrak{\Omega}_{i}^{b} \|\left(n_{j}^{\prime}\right)$ for all $j$ for $i=1, \cdots, r$. By renumbering the $n_{j}$, we may assume that $\mathfrak{D}_{r}^{b} r \|\left(n_{1}\right)$. Choose $u \in \mathfrak{Q}_{1^{b_{1}+1}}^{b_{1}} \ldots \mathfrak{\Omega}_{r-1}^{b} r_{-1}^{+1}, u \notin \mathfrak{Q}_{r}$, and replace each $n_{j}$ for which $n_{j} \in \mathfrak{D}_{r}^{b_{r}+1}$ by $n_{j}^{\prime}=n_{j}+u n_{1}$. Then

$$
\begin{gathered}
n_{j}^{\prime} \equiv n_{j}\left(\bmod \mathfrak{D}_{i}^{b_{i}+1}\right), \\
n_{j}^{\prime} \equiv u n_{1} \text { or } n_{j}\left(\bmod \mathfrak{Q}_{r}^{b^{+}+1}\right),
\end{gathered}
$$

${ }^{1} Z$ denotes the set of rational integers. 
according as to whether $n_{j} \in \mathfrak{S}_{r}^{b} r^{+1}$ or not. This implies the desired result.

3. Since the group $H$ properly contains $G(\mathfrak{M N})$, by the preceding discussion it follows that there exists an element

$$
A=\left(\begin{array}{ll}
a & b \\
c & d
\end{array}\right) \in H
$$

in which $(c)=\mathfrak{M}(5$ with $((\mathfrak{S}, 9) i)=(1)$. Therefore $H$ also contains, for each $x \in \mathscr{D}$, the product

$$
\left(\begin{array}{ll}
1 & x \\
0 & 1
\end{array}\right)\left(\begin{array}{ll}
a & b \\
c & d
\end{array}\right)=\left(\begin{array}{cc}
a+x c & \cdot \\
c & .
\end{array}\right)
$$

Since $(a, c)=(1)$, we may choose $x$ so that $((a+x c)$, $\mathfrak{M})=(1)$. Hence we may assume that $H$ contains an element $A$ given by (5), satisfying

$$
((a), \mathfrak{M})=(1), \quad(c)=\mathfrak{N}(\mathfrak{S}, \quad(\mathfrak{S}, \mathfrak{M})=(1) .
$$

Next we remark that

$$
\left(\begin{array}{cc}
1 & 0 \\
n_{j} y_{j} & 1
\end{array}\right)\left(\begin{array}{ll}
a & b \\
c & d
\end{array}\right)=\left(\begin{array}{cc}
\cdot & \cdot \\
n_{j} y_{j} a+c & .
\end{array}\right)
$$

so that if $y_{j}$ is chosen to satisfy $n_{j} y_{j} a+c \in \mathfrak{M} \mathfrak{R}$, it will follow that

$$
W_{j}^{*}=\left(\begin{array}{cc}
1 & 0 \\
n_{j} y_{j} & 1
\end{array}\right) \in H \text {. }
$$

But now $n_{j} y_{j} a+c \in \mathfrak{M} \Re$ means that

$$
n_{j} y_{j} a \equiv-c \quad\left(\operatorname{mad} \mathfrak{\Omega}_{i}^{a_{i}+b_{i}}\right) \text { for each } i .
$$

If $a_{i}=0$, then $\Omega_{i}^{b_{i}}$ divides both $\left(n_{j}\right)$ and $(c)$, and there is no condition on $y_{j}$. If $a_{i}>0$, then ord $\mathfrak{a}_{i} a=0$, ord $\mathfrak{a}_{i} n_{j}=b_{i}$, and ord $\mathfrak{a}_{i} c=b_{i}$, so that the congruence is solvable for $y_{j}$. We remark further that $y_{j}$ may be chosen coprime to any fixed ideal. Thus for each $n_{j}$, there exists an element $y_{j} \in \mathscr{D}$ such that $\left(\left(y_{j}\right), \mathfrak{M} \mathfrak{R}\right)=(1)$, and for which $W_{j}^{*} \in H$. In order to complete the proof of the theorem, we need only show that if

$$
\left(\begin{array}{cc}
1 & 0 \\
n y & 1
\end{array}\right) \in H \text {. }
$$

where $\mathfrak{Q}_{i}^{b} \|(n)$ for each $i$, and where $((y), \mathfrak{M} \mathfrak{R})=(1)$, then also

$$
\left(\begin{array}{ll}
1 & 0 \\
n & 1
\end{array}\right) \in H
$$

4. Now let $d \in \mathscr{D}$ be any element coprime to $\mathfrak{M} \mathfrak{R}$; then there 
exists $a \in \mathbb{Z}$ such that $a d \equiv 1(\bmod \mathfrak{M M})$. Setting $a d=1+c$, with $c \in \mathfrak{M N}$, we see that

$$
\left(\begin{array}{ll}
a & 1+x a \\
c & d+x c
\end{array}\right) \in G(\mathfrak{M N}) \subset H
$$

for all $x \in \mathscr{D}$. In particular, choosing $x$ so that $1+x a \in \mathfrak{M} \mathscr{N}$, we see that $H$ contains a matrix

$$
\left(\begin{array}{ll}
a & b \\
c & d
\end{array}\right)
$$

in which both $b$ and $c$ lie in $\mathfrak{M E} \Re$. Therefore $H$ also contains

$$
\left(\begin{array}{ll}
a & b \\
c & d
\end{array}\right)\left(\begin{array}{cc}
1 & 0 \\
n y & 1
\end{array}\right) \equiv\left(\begin{array}{cc}
a & \cdot \\
d y n & .
\end{array}\right)(\bmod \mathfrak{M N})
$$

But then

$$
\left(\begin{array}{cc}
1 & 0 \\
n u & 1
\end{array}\right)\left(\begin{array}{cc}
a & \cdot \\
d y n & .
\end{array}\right)=\left(\begin{array}{cc}
\cdot & \cdot \\
n(u a+d y) & .
\end{array}\right)
$$

and so if $u \in \mathscr{O}$ is chosen satisfying $n(u a+d y) \in \mathfrak{M} \mathfrak{N}$, then the matrix

$$
\left(\begin{array}{cc}
1 & 0 \\
n u & 1
\end{array}\right) \in H
$$

However, $n(u a+d y) \in \mathfrak{M N}$ if and only if

$$
n(u a+d y) \equiv 0 \quad\left(\bmod \mathfrak{O}_{i}^{a^{+}+b_{i}}\right) \text { for each } i,
$$

that is,

$$
u \equiv-d y / a \quad\left(\bmod \Omega_{i}^{a_{i}}\right) \quad \text { for each } i .
$$

Since $a d \equiv 1\left(\bmod \mathfrak{Q}_{i}^{a}\right)$, this gives $u \equiv-y d^{2}\left(\bmod \mathfrak{D}_{i}^{a_{i}}\right)$.

We have thus shown that (8) implies

$$
\left(\begin{array}{cc}
1 & 0 \\
n y d^{2} & 1
\end{array}\right) \in H
$$

for every $d$ coprime to $M \&$ R. Consequently $H$ contains every product of such matrices, that is,

$$
\left(\begin{array}{cc}
1 & 0 \\
n y x & 1
\end{array}\right) \in H
$$

whenever $x=\sum d_{i}^{2}$, with each $d_{i}$ coprime to $\mathfrak{M} \Re$. To complete the proof, it suffices to show that there exists such an $x$ for which $y x \equiv 1$ $(\bmod \mathfrak{M})$. For then $y x=1+m$ with $m \in \mathfrak{M}$, and 


$$
\left(\begin{array}{cc}
1 & 0 \\
n y x & 1
\end{array}\right)\left(\begin{array}{cc}
1 & 0 \\
-m n & 1
\end{array}\right)=\left(\begin{array}{ll}
1 & 0 \\
n & 1
\end{array}\right) \in H
$$

Finally, $y \sum d_{i}^{2} \equiv 1(\bmod \mathfrak{M})$ implies $y=\sum\left(y d_{i}\right)^{3}(\bmod \mathfrak{M})$, and conversely, so we need only show the existence of numbers $d_{i}$ coprime to MiN such that

$$
y \equiv \sum d_{i}^{2} \quad\left(\bmod \mathfrak{M}_{i}\right)
$$

5. We begin by examining the finite field $\mathscr{L}=\mathscr{C} / \Sigma_{i}$, where $\Omega_{i} \mid \mathfrak{M}$. The characteristic of $\mathbb{Z}$ is $p$, the unique rational prime in $\mathfrak{I}_{i}$. Since $(M,(6))=(1)$, certainly $p>3$. The nonzero elements of $\mathscr{I}$ form a multiplicative group, and the map $x \rightarrow x^{2}$ gives an endomorphism of this group with kernel \pm 1 . Hence exactly half of the nonzero elements of $\mathfrak{R}$ are squares, and we have the usual rules for multiplying quadratic residues and nonresidues. In particular, $\&$ contains at least two distinct squares.

Now we show that $\mathfrak{M}$ is a power of a single prime. For let $\mathfrak{Z}_{1} \mid \mathfrak{M}$, $\mathfrak{O}_{2} \mid \mathfrak{M}$, with $\mathfrak{I}_{1} \neq \mathfrak{O}_{2}$, and let $p$ be the characteristic of $\_/ \mathfrak{I}_{1}$. If we choose $d_{i} \equiv 1\left(\bmod \mathfrak{I}_{1}\right)(i=1, \cdots, p)$, then $x=\sum d_{i}^{2} \equiv 0\left(\bmod \mathfrak{I}_{1}\right)$. On the other hand, since there are at least two distinct squares $\left(\bmod \mathfrak{D}_{2}\right)$ by the above argument, we may choose the $d_{i}$ coprime to $99 x$ such that $\left(\Omega_{2},(x)\right)=(1)$. But then the matrix given by (9) lies in $H$, which is impossible by virtue of the discussion at the end of Part 1 of this proof, for $\mathfrak{Q}_{1} \mathfrak{M} \mid((n y x), \mathfrak{M} \mathfrak{M})$, but $\mathfrak{\Omega}_{2} \nmid((n y x), \mathfrak{M} \mathfrak{N})$.

We need only prove now that

$$
y \equiv \sum d_{i}^{2} \quad\left(\bmod \Omega^{\prime \prime}\right)
$$

is solvable for a set of $d_{i}$ each coprime to $\cong$. Since $(\Omega,(2))=(1)$, we may show by a well-known procedure that for any $d$ coprime to $\Omega$, and any $q \in \mathfrak{\Omega}$, there exists $\bar{d} \in \mathscr{O}$ such that

$$
d^{2}+q \equiv \overline{d^{2}} \quad\left(\bmod \Omega^{a}\right)
$$

Hence it suffices to prove that

$$
y \equiv \sum d_{i}^{2} \quad(\bmod \Omega)
$$

is solvable, that is, that every nonzero element in $\mathscr{L}=\mathscr{C} / \mathfrak{Q}$ is expressible as a sum of squares. We need only show that any non-square in $\mathbb{Z}$ is a sum of squares, and for this it is sufficient to show that at least one non-square is the sum of squares. For then all non-squares are obtained from the given non-square by multiplying by suitable squares.

Now if the sum of squares in $\&$ were always a square, then the squares would form a subfield $\mathfrak{K}$, and we would have $[\mathfrak{R}: \mathfrak{K}]=2$. This 
is impossible, since the number of elements in $\mathscr{L}=\mathscr{D} / \mathfrak{Q}$ is odd, being a power of the characteristic of $\mathscr{D} / \mathfrak{D}$.

3. Two examples. The hypothesis that $(\mathfrak{M},(6))=(1)$ seems almost superfluous in the above proof, entering only in the discussion of squares $\left(\bmod \mathfrak{D}_{i}^{a_{i}}\right)$, and it might be thought that a different proof could be found which would obviate this restriction. To show that this is not the case, we give here two examples in which (2) holds, but where the conclusion of Theorem 1 is not valid; in the first example, (M, (2)) $\neq(1)$, and in the second, $(\mathfrak{M},(3)) \neq(1)$. We shall use the notation $G(n)$ to denote $G((n))$, where $(n)$ is a principal ideal.

Firstly, let $\mathscr{O}$ be the ring of Gaussion integers. We shall exhibit a group $H$ for which

$$
G(4) \subset H \subset G(2)
$$

with both inclusions proper, and such that $H \neq G(2+2 i)$. Since $G(2+2 i)$ is the only congruence subgroup between $G(2)$ and $G(4)$, this shows that the conclusion of Theorem 1 does not hold here.

Let us set

$$
B=\left(\begin{array}{ll}
1 & 0 \\
2 & 1
\end{array}\right)
$$

Let $H$ be the group generated by $B$ and $G(4)$; clearly (10) holds. Let $X$ denote the general element of $G(4)$, say

$$
X=\left(\begin{array}{ll}
a & b \\
c & d
\end{array}\right) \equiv\left(\begin{array}{ll}
a & b \\
0 & d
\end{array}\right) \quad(\bmod 4) \text {. }
$$

Then

$$
B X B^{-1} \equiv\left(\begin{array}{ll}
1 & 0 \\
2 & 1
\end{array}\right)\left(\begin{array}{ll}
a & b \\
0 & d
\end{array}\right)\left(\begin{array}{ll}
1 & 0 \\
2 & 1
\end{array}\right)=\left(\begin{array}{cc}
\cdot & \cdot \\
2(a+d) & .
\end{array}\right) \quad(\bmod 4) .
$$

However, $a d \equiv 1(\bmod 4)$ implies (by consideration of cases) that $a+d \equiv 0$ $(\bmod 2)$. Therefore $B G(4) B^{-1}=G(4)$, and hence $G(4)$ is of index 2 in $H$. Since $G(4)$ has index 4 in $G(2)$, we see that $H$ is neither $G(4)$ nor $G(2)$. Furthermore, $H \neq G(2+2 i)$, since $B \notin G(2+2 i)$.

For our second example, let us take $\mathscr{D}$ to be the ring of integers in the field obtained by adjoining $\alpha$ to the rational field, where $\alpha$ is a zero of $x^{2}+5$. Then $\mathscr{D}=\{a+b \alpha: a \in Z, b \in Z\}$. In this ring we have the factorization $(3)=\mathfrak{Z}_{1} \mathfrak{\Omega}_{2}$, where

$$
\mathfrak{\Omega}_{1}=(3,2+\alpha), \quad \mathfrak{D}_{2}=(3,2-\alpha)
$$

are prime ideals with norm 3 . Let $H$ be the group generated by $B$ and $G(9)$, where 


$$
B=\left(\begin{array}{ll}
1 & 0 \\
3 & 1
\end{array}\right) \text {. }
$$

Then clearly $G(9) \subset H \subset G(3)$, with the first inclusion proper. Certainly $H$ is not an intermediate congruence subgroup, since $H=G(3 \Re)$ would imply (because $B \in H)$ that $3 \mathfrak{P} \mid(3)$. We need only prove that $H \neq G(3)$, which is the case since $[H: G(9)]=3$, whereas $[G(3): G(9)]=9$. For we have, as in the previous example, the result that $B G(9) B^{-1}=G(9)$, following easily from the fact that $a d \equiv 1(\bmod 9)$ implies $a \equiv d(\bmod 3)$.

The question as to the necessary and sufficient conditions on the ideals $\mathfrak{M}, \mathfrak{N}$ to insure the validity of the conclusion of Theorem 1 seems more difficult, since the answer will certainly depend on the structure of $\mathscr{C}$. For example, it is possible to give certain special cases in which Theorem 1 holds, even though $(\mathfrak{M},(6)) \neq(1)$.

4. Higher dimension. Turning now to the domain of rational integers, there is no direct and complete generalization of Newman's theorem valid for higher dimensions, owing to the profusion of possible congruence subgroups. For example, we may define a 3-parameter family of subgroups $G(m, n, r)$ of $M_{3}^{+}$, where $r \mid(m, n)$, by setting

$$
G(m, n, r)=\left\{\left(a_{i j}\right) \in M_{3}^{+}: m\left|a_{21}, n\right| a_{32}, r[m, n] \mid a_{31}\right\} .
$$

(Here, $[m, n]=$ L.C.M. of $m$ and $n$.) It would be reasonable to conjecture that if $H$ is a group satisfying $G(a m, b n, c r) \subset H \subset G(m, n, r)$, where $c r \mid(a m, b n)$, then $H=G(\alpha m, \beta n, \gamma r)$ with $\alpha|a, \beta| b, \gamma \mid c$, and $\gamma r \mid(\alpha m, \beta n)$. The proof of such a conjecture would be rather tedious, and would have no direct generalization to higher dimensions.

We shall therefore restrict our attention to two specific types of groups which are readily defined in all dimensions, the column groups

$$
C_{m}=\left\{\left(a_{i j}\right) \in M_{r}^{+}: m\left|a_{21}, m\right| a_{31}, \cdots, m \mid a_{r 1}\right\}
$$

and the row groups

$$
R_{n}=\left\{\left(a_{i j}\right) \in M_{r}^{+}: n\left|a_{r 1}, n\right| a_{r 2}, \cdots, n \mid a_{r, r-1}\right\} .
$$

THEOREM 2." Let $H$ be a subgroup of $M_{r}^{+}$satisfying

$$
\left(C_{a m} \cap R_{b n}\right) \subset H \subset\left(C_{m} \cap R_{n}\right),
$$

where $(a m, b n)=1$. Then $H=C_{\alpha m} \cap R_{\beta n}$, where $\alpha \mid a$ and $\beta \mid b$.

Proof. We shall carrry out the proof for $r=3$, since higher dimen-

$2 \mathrm{M}$. Newman has informed the authors that he has independently obtained the results comprised in this theorem. 
sions present little additional difficulty. We shall use induction on the number of prime factors of $b$, and begin with the case $b=1$. When $a=1$ also, the result is clear. Suppose it is true for all $a$ with fewer than $k$ prime factors, and now let $a$ have $k$ prime factors. Then by hypothesis

$$
\left(C_{a m} \cap R_{n}\right) \subset H \subset\left(C_{m} \cap R_{n}\right) .
$$

As in the proof of Theorem 1, intersect this with $C_{A m}$, where $A \neq 1$ and $A \mid a$. Then either the desired result follows from the induction hypothesis on $a$, or else for each such $A$ we have

$$
H \cap C_{A m}=C_{a m} \cap R_{n} \text {. }
$$

Therefore for any element

$$
T=\left(\begin{array}{lll}
a_{11} & \cdot & \cdot \\
m a_{21} & \cdot & \cdot \\
m n a_{31} & \cdot & \cdot
\end{array}\right)
$$

of $H$, either $\left(a_{21}, a_{31}, a\right)=1$ or $\left(a_{21}, a_{31}, a\right)=a$.

Now suppose that $H \neq C_{a m} \cap R_{n}$. Then for some $T \in H$ the former alternative occurs. Then for any $x, y, z$, the matrix

$$
T_{1}=\left(\begin{array}{lll}
1 & x & y \\
0 & 1 & z \\
0 & 0 & 1
\end{array}\right)\left(\begin{array}{lll}
a_{11} & . & . \\
m a_{21} & . & . \\
m n a_{31} & . & .
\end{array}\right)=\left(\begin{array}{lll}
a_{11}+m\left(x a_{21}+y n a_{31}\right) & . & . \\
m\left(a_{21}+z n a_{31}\right) & . & . \\
m n a_{31} & . & .
\end{array}\right)
$$

lies in $H$. Since $\left(a_{11}, m a_{21}, m n a_{31}\right)=1$, by proper choice of $x, y, z$ we may make the elements in the $(1,1)$ and $(2,1)$ positions of $T_{1}$ coprime to $a$. Changing notation, we may now assume that $H$ contains an element $T$ given by (11), satisfying $\left(a_{11}, a\right)=\left(a_{21}, a\right)=1$.

Next, replacing $T$ by

$$
\left(\begin{array}{ccc}
1 & 0 & 0 \\
0 & 1 & 0 \\
0 & n t & 1
\end{array}\right) T \in H
$$

leaves $a_{11}$ and $a_{21}$ unaltered, and replaces $a_{31}$ by $a_{31}^{\prime}=a_{31}+t a_{21}$. By proper choice of $t$, we may make $\left(a_{31}^{\prime}, a\right)=1$. Again changing notation, $H$ now contains an element $T$ for which

$$
\left(a_{11}, a\right)=\left(a_{21}, a\right)=\left(a_{31}, a\right)=1 .
$$

We next observe that 


$$
\left(\begin{array}{ccc}
1 & 0 & 0 \\
m y & 1 & 0 \\
m n z & 0 & 1
\end{array}\right) T=\left(\begin{array}{lll}
a_{11} & \cdot \\
m\left(a_{11} y+a_{21}\right) & . & . \\
m n\left(a_{11} z+a_{31}\right) & . & .
\end{array}\right)
$$

If $y$ and $z$ are chosen so that

$$
a_{11} y+a_{21} \equiv a_{11} z+a_{31} \equiv 0 \quad(\bmod a),
$$

then the right-hand side of (12) will lie in $C_{a m} \cap R_{n}$, hence in $H$. Therefore $H$ contains a matrix

$$
\left(\begin{array}{lll}
1 & 0 & 0 \\
m y & 1 & 0 \\
m n z & 0 & 1
\end{array}\right)
$$

with both $y$ and $z$ coprime to $a$.

But then

$$
\left(\begin{array}{lll}
1 & 0 & 0 \\
m a u & 1 & t \\
0 & 0 & 1
\end{array}\right)\left(\begin{array}{lll}
1 & 0 & 0 \\
m y & 1 & 0 \\
m n z & 0 & 1
\end{array}\right)\left(\begin{array}{ccr}
1 & 0 & 0 \\
0 & 1 & -t \\
0 & 0 & 1
\end{array}\right)=\left(\begin{array}{ccc}
1 & 0 & 0 \\
m(a u+y+n t z) & 1 & 0 \\
m n z & 0 & 1
\end{array}\right) \in H,
$$

and also

$$
\left(\begin{array}{ccc}
1 & 0 & 0 \\
0 & 1 & 0 \\
\text { mnau } & n t & 1
\end{array}\right)\left(\begin{array}{lll}
1 & 0 & 0 \\
m y & 1 & 0 \\
m n z & 0 & 1
\end{array}\right)\left(\begin{array}{ccc}
1 & 0 & 0 \\
0 & 1 & 0 \\
0 & -n t & 1
\end{array}\right)=\left(\begin{array}{ccc}
1 & 0 & 0 \\
m y & 1 & 0 \\
m n(a u+t y+z) & 0 & 1
\end{array}\right) \in I I .
$$

By proper choice of $t$ and $u$ in each case, we deduce that $P^{v} \in H$ and $S^{z} \in H$, where

$$
P=\left(\begin{array}{lll}
1 & 0 & 0 \\
m & 1 & 0 \\
0 & 0 & 1
\end{array}\right), \quad S=\left(\begin{array}{ccc}
1 & 0 & 0 \\
0 & 1 & 0 \\
m n & 0 & 1
\end{array}\right)
$$

On the other hand, $P^{a} \in H$ and $S^{a} \in H$. Since $(y, a)=(z, a)=1$, it follows at once that $P$ and $S$ are both in $H$.

Now let

$$
U=\left(\begin{array}{lll}
1 & 1 & 0 \\
0 & 1 & 0 \\
0 & 0 & 1
\end{array}\right), \quad V=\left(\begin{array}{lll}
1 & 0 & 1 \\
0 & 1 & 0 \\
0 & 0 & 1
\end{array}\right)
$$

Then $P, S, U, V$ generate a complete set of left coset representatives of $C_{m} \cap R_{n}$ with respect to $C_{a m} \cap R_{n}$. Since $P, S, U, V$ are all in $H$, this shows that $H=C_{m} \cap R_{n}$. Hence we have established the theorem 
when $b=1$, for all $a$. A corresponding proof can be given when $a=1$, for all $b$.

Suppose now that the theorem has been proved for all $a$ whenever $b$ has fewer than $k$ prime factors, and take $b$ to have $k$ prime factors. The result certainly holds when $a=1$. Assume it true for $a$ having fewer than $k^{\prime}$ prime factors, and now let $a$ have $k^{\prime}$ prime factors. By hypothesis

$$
\left(C_{a m} \cap R_{b n}\right) \subset H \subset\left(C_{m} \cap R_{n}\right) .
$$

Intersect this with $C_{A m}$, where $A \mid a$ and $A \neq 1$. Then as before, the result holds (by virtue of the induction hypothesis) unless

$$
H \cap C_{A m}=C_{a m} \cap R_{b n}
$$

for all such $A$. Likewise, the result holds unless for each $B \neq 1, B \mid b$ we have

$$
H \cap R_{B n}=C_{a m} \cap R_{b n}
$$

From (13) and (14) we deduce that if

$$
T=\left(\begin{array}{ccc}
a_{11} & a_{12} & a_{13} \\
m a_{21} & a_{22} & a_{23} \\
m n a_{31} & n a_{32} & a_{33}
\end{array}\right) \in H,
$$

then either

$$
\left(a_{21}, a_{31}, a\right)=a \quad \text { and } \quad\left(a_{31}, a_{32}, b\right)=b
$$

or

$$
\left(a_{21}, a_{31}, a\right)=\left(a_{31}, a_{32}, b\right)=1 \text {. }
$$

To complete the proof, we shall assume that $H \neq C_{a m} \cap R_{b n}$, and obtain a contradiction. Under this hypothesis, (16) must hold for some $T \in H$. Replacing $T$ by

$$
\left(\begin{array}{lll}
1 & x & y \\
0 & 1 & z \\
0 & 0 & 1
\end{array}\right) T
$$

with suitably chosen $x, y, z$, we may hereafter assume that $\left(a_{11}, a b\right)=$ $\left(a_{21}, a\right)=1$.

We now show that $H$ contains a matrix $T$ satisfying

$$
\left(a_{11}, a b\right)=\left(a_{21}, a\right)=\left(a_{22}, b\right)=1, \quad b \mid a_{12} .
$$

Upon replacing $T$ of the preceding paragraph by 


$$
T\left(\begin{array}{lll}
1 & k & 0 \\
0 & 1 & 0 \\
0 & 0 & 1
\end{array}\right)
$$

$a_{11}$ and $a_{21}$ are unchanged, and $a_{12}$ becomes $a_{12}^{\prime}=a_{12}+k a_{11}$. If we choose $k$ so that $b \mid a_{12}^{\prime}$, we shall have a matrix (again denoted by $T$ ) in $H$, for which $\left(a_{11}, a b\right)=\left(a_{21}, a\right)=1, b \mid a_{12}$. Lastly, replace $T$ by

$$
\left(\begin{array}{rrr}
1 & 0 & 0 \\
0 & 1 & a x \\
0 & 0 & 1
\end{array}\right) T \in H
$$

This leaves $a_{11}$ and $a_{12}$ unaltered, does not change $a_{21}(\bmod a)$, and replaces $a_{22}$ by $a_{22}^{\prime}=a_{22}+a n x a_{32}$. Since $\left(a_{22}, a n a_{32}, b\right)=1$, we may choose $x$ so that $\left(a_{22}^{\prime}, b\right)=1$. This new matrix $T$ will then satisfy (17).

Let us put

$$
Y=\left(\begin{array}{ccc}
1 & 0 & 0 \\
m x & 1 & 0 \\
m n y & n z & 1
\end{array}\right)
$$

Then

$$
Y T=\left(\begin{array}{lcc}
a_{11} & \cdot & \cdot \\
m\left(a_{21}+x a_{11}\right) & \cdot & \cdot \\
m n\left(a_{31}+y a_{11}+z a_{21}\right) & n\left(a_{32}+m y a_{12}+z a_{22}\right) & .
\end{array}\right) .
$$

If $x, y, z$ satisfy

$$
\begin{array}{cl}
a_{21}+x a_{11} \equiv 0 & (\bmod a), \\
a_{31}+y a_{11}+z a_{21} \equiv 0 & (\bmod a b), \\
a_{32}+m y a_{12}+z a_{22} \equiv 0 & (\bmod b),
\end{array}
$$

then the product $Y T$ will lie in $C_{a m} \cap R_{b n}$, hence in $H$, and so $Y \in H$. We may certainly solve $(18)$ with $(x, a)=1$. Since $b \mid a_{12}$, we may solve (20) for $z(\bmod b)$. Fixing $z$ arbitrarily $(\bmod a)$, we may then solve (19) for $y$, since $\left(a_{11}, a b\right)=1$. Therefore $H$ contains a matrix $Y$ in which $(x, a)=1$.

But now

$$
Y^{b}\left(\begin{array}{ccc}
1 & 0 & 0 \\
0 & 1 & 0 \\
0 & -b n z & 1
\end{array}\right)=\left(\begin{array}{ccc}
1 & 0 & 0 \\
m b x & 1 & 0 \\
m n y_{1} & 0 & 1
\end{array}\right) \in H
$$

and so 


$$
W=\left(\begin{array}{ccc}
1 & 0 & 0 \\
m b x & 1 & 0 \\
m n y_{1} & 0 & 1
\end{array}\right)^{b}=\left(\begin{array}{ccc}
1 & 0 & 0 \\
m b^{2} x & 1 & 0 \\
m n b y_{1} & 0 & 1
\end{array}\right) \in H
$$

However, $W \in R_{b n}$, so by (14) since $b>1$, also $W \in C_{a m}$. This is impossible, since $\left(a, b^{2} x\right)=1$. We thus have a contradiction, and so $H$ must equal $C_{a m} \cap R_{b n}$. This comptes the proof.

We remark in conclusion that various special theorems may be proved by similar methods. For example, using the notation at the beginning of this section, we may show that $G(m, n, r s) \subset H \subset G(m, n, s)$ implies $H=G(m, n, t s)$ with $t \mid r$.

\section{REFERENCE}

1. Morris Newman, Structure theorems for modular subroups, Duke Math. J., 22 (1955), 25-32,

INSTITUTE FOR ADVANCED STUDY; UNIVERSITY OF ILLINOIS.

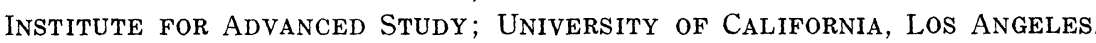




\section{PACIFIC JOURNAL OF MATHEMATICS}

\section{EDITORS}

\author{
H. L. Royden \\ Stanford University \\ Stanford, California \\ E. Hewitt \\ University of Washington \\ Seattle 5 , Washington
}

R. P. Dilworth

California Institute of Technology

Pasadena 4, California

\author{
A. HorN* \\ University of California \\ Los Angeles 24, California
}

\section{ASSOCIATE EDITORS}

\author{
E. F. BECKENBACH \\ C. E. BURGESS \\ H. BUSEMANN \\ H. FEDERER
}

\author{
M. HALL \\ P. R. HALMOS \\ V. GANAPATHY IYER \\ R. D. JAMES
}

\author{
M. S. KNEBELMAN \\ I. NIVEN \\ T. G. OSTROM \\ M. M. SCHIFFER
}

J. J. STOKER

G. SZEKERES

F. WOLF

K. YOSIDA

\section{SPONSORS}

\author{
UNIVERSITY OF BRITISH COLUMBIA \\ CALIFORNIA INSTITUTE OF TECHNOLOGY \\ UNIVERSITY OF CALIFORNIA, BERKELEY \\ UNIVERSITY OF CALIFORNIA, DAVIS \\ UNIVERSITY OF CALIFORNIA, LOS ANGELES \\ UNIVERSITY OF CALIFORNIA, SANTA BARBARA \\ MONTANA STATE UNIVERSITY \\ UNIVERSITY OF NEVADA \\ OREGON STATE COLLEGE \\ UNIVERSITY OF OREGON \\ UNIVERSITY OF SOUTHERN CALIFORNIA
}

STANFORD RESEARCH INSTITUTE

STANFORD UNIVERSITY

UNIVERSITY OF UTAH

WASHINGTON STATE COLLEGE

UNIVERSITY OF WASHINGTON

AMERICAN MATHEMATICAL SOCIETY HUGHES AIRCRAFT COMPANY SHELL DEVELOPMENT COMPANY

Mathematical papers intended for publication in the Pacific Journal of Mathematics should be typewritten (double spaced), and the author should keep a complete copy. Manuscripts may be sent to any of the editors. Manuscripts intended for the outgoing editors should be sent to their successors. All other communications to the editors should be addressed to the managing editor, Alfred Horn at the University of California, Los Angeles 24, California.

50 reprints of each article are furnished free of charge; additional copies may be obtained at cost in multiples of 50 .

The Pacific Journal of Mathematics is published quarterly, in March, June, September, and December. The price per volume (4 numbers) is $\$ 12.00$; single issues, $\$ 3.50$. Back numbers are available. Special price to individual faculty members of supporting institutions and to individual members of the American Mathematical Society: $\$ 4.00$ per volume; single issues, $\$ 1.25$.

Subscriptions, orders for back numbers, and changes of address should be sent to Pacific Journal of Mathematics, c/o University of California Press, Berkeley 4, California.

Printed at Kokusai Bunken Insatsusha (International Academic Printing Co., Ltd.), No. 10, 1-chome, Fujimi-cho, Chiyoda-ku, Tokyo, Japan.

* During the absence of E. G. Straus.

PUBLISHED BY PACIFIC JOURNAL OF MATHEMATICS, A NON-PROFIT CORPORATION COPYRIGHT 1956 BY PACIFIC JOURNAL OF MATHEMATICS 


\section{Pacific Journal of Mathematics}

\section{Vol. 6, No. $3 \quad$ BadMonth, 1956}

Richard Arens and James Eells, Jr., On embedding uniform and topological spaces ............................................ 397

N. Aronszajn and Prom Panitchpakdi, Extension of uniformly continuous transformations and hyperconvex metric spaces .............. 405

Kai Lai Chung and Cyrus Derman, Non-recurrent random walks ........ 441

Harry Herbert Corson, III, On some special systems of equations . . . . . . . . . 449

Charles W. Curtis, On Lie algebras of algebraic linear transformations . . . 453

Isidore Heller, Neighbor relations on the convex of cyclic permutations . . . . 467

Solomon Leader, Convergence topologies for measures and the existence of transition probabilities..................................... 479

D. H. Lehmer, On certain character matrices ...................... 491

Michael Bahir Maschler, Minimal domains and their Bergman kernel function ........................................... 501

Wm. M. Myers, Functionals associated with a continuous transformation ................................... 517

Irving Reiner and Jonathan Dean Swift, Congruence subgroups of matrix groups ....................................... 529

Andrew Sobczyk, Simple families of lines ................... 541

Charles Standish, A class of measure preserving transformations ........ 553 Jeremiah Milton Stark, On distortion in pseudo-conformal mapping ..... 565 